\title{
Learning curve of laparoscopic Kasai portoenterostomy for biliary atresia: report of 100 cases
}

\author{
Yi Ji', Kaiying Yang ${ }^{1}$, Xuepeng Zhang ${ }^{2}$, Siyuan Chen $^{2}$ and Zhicheng $\mathrm{Xu}^{{ }^{1 *}}$ (D)
}

\begin{abstract}
Background: Laparoscopic Kasai portoenterostomy (LKPE) is performed for biliary atresia (BA). As LKPE is a technically demanding operation, a learning curve should be defined to guide training. The aim of this study was to identify the learning curve of LKPE for BA.

Methods: Metrics of perioperative safety and efficiency for 100 cases of LKPE were evaluated. Outcomes were followed to $67.2 \pm 12.1$ months. Cumulative sum (CUSUM) analysis was used to identify inflexion point corresponding to the learning curve. Outcome measures included operative time (ORT), rate of clearance of jaundice (CJ) and survival with native liver (SNL).

Results: Between May 2009 and May 2013, 100 consecutive patients with BA underwent LKPE. The rate of conversion from LKPE to open Kasai portoenterostomy (OKPE), intraoperative transfusion and any perioperative complications was 11,26 and 16\%, respectively. There was no perioperative mortality. The CUSUM analysis revealed a learning curve of 50 for LKPE. Precipitous ORT reductions from an initial mean operative time of $316.3 \mathrm{~min}$ that was observed in the first 50 to $232.2 \mathrm{~min}$ of the late 50 cases $(P<0.01)$. Subsequently, cases 1 to 50 were considered 'early experience', whereas cases 51 and higher were considered as 'late experience' for statistical analysis. The rate of CJ and SNL was significantly higher after the early 50 cases $(P<0.05)$. In contrast, the rate of intraoperative transfusion, the median time of oral feeding initiated after operation, and the length of hospital stay was not different between the both groups $(P>0.05)$.

Conclusions: In this experience, improved perioperative and postoperative parameters for LKPE were observed in the last 50 patients when compared with the first 50 patients. The dedicated training is likely to contribute to significantly shorter learning curves in future adopters.
\end{abstract}

Keywords: Biliary atresia, Portoenterostomy, Laparoscopy, Learning curve

\section{Background}

Laparoscopic Kasai portoenterostomy (LKPE) has first been applied to biliary atresia (BA) by Esteves in 2002 [1]. Previously, LKPE had not been showed to be as efficacious as the open Kasai portoenterostomy (OKPE) [2-4]. However, recent reports revealed that the 3- and 5 -year survivals with native liver (SNL) after LKPE were not inferior to that after OKPE $[5,6]$. Similar to other laparoscopic procedures, a learning curve may exist for LKPE, which, if identified, may allow new adopters to

\footnotetext{
*Correspondence: xzcxewk@163.com

${ }^{1}$ Department of Pediatric Surgery, West China Hospital of Sichuan University,

Chengdu, Sichuan, \#37 Guo-Xue-Xiang, Chengdu 610041, China

Full list of author information is available at the end of the article
}

improve their insight. For LKPE, the learning curve may entail the mastery of key points of this specific procedure. These key points include optimal port placement, the full exposure of porta hepatis, the appropriate level of resection of fibrous cone, the demanding technique of portoenterostomy, the development of close coordination between the console surgeon and bedside assistant, and the overcoming of the loss of tactile feedback.

The present paper reports the clinical data of the first 100 cases of LKPE, which were accomplished at our hospital, with the purpose of discerning major inflexion points and landmarks in the optimization of perioperative outcomes.

(C) The Author(s). 2018 Open Access This article is distributed under the terms of the Creative Commons Attribution 4.0 International License (http://creativecommons.org/licenses/by/4.0/), which permits unrestricted use, distribution, and 


\section{Methods}

\section{Design and study population}

The first 100 consecutive cases of LKPE were reviewed retrospectively. All operations were carried out by the same surgical team with substantial laparoscopic experiences at West China Hospital of Sichuan University between May 2009 and May 2013. Approval was obtained from the West China Hospital of Sichuan University Institutional Review Board. All procedures followed the research protocols approved by Sichuan University and West China Hospital of Sichuan University and was conducted according to the Declaration of Helsinki. Written informed consent was provided by the patients' parents for their clinical records to be used in this study.

\section{Operative technique}

The operative technique of LKPE has already been described in a previous study from our group [7]. Briefly, a percutaneous suture was used to snare the round and falciform ligament and retract the liver. Afterward, another two percutaneous transhepatic sutures were introduced individually into the left lobe and right lobe for better hilar exposure. The atretic gallbladder and cystic duct were dissected free from the gall bladder fossa. The distal part of the extrahepatic duct was divided behind the duodenum with $5 \mathrm{~mm}$ monopolar hook. After the base of the fibrous cone was reached, the fibrous cord was transected carefully with laparoscopic scissors. The level of resection of fibrous cone was depended on the appearance of bile juice secreted at the fibrous stump. Bleeding from the fibrous remains of portal plate was controlled by direct pressure with moist gauze. Next, a 25-cm Roux-en-Y limb was fashioned and delivered to the hilum through a retro-colic path after the pneumoperitoneum was re-established.

\section{Definitions and postoperative management}

ORT was calculated as the length of time between skin incision and closure. Perioperative complications were complications that occurred during the perioperative period. Theses perioperative complications included wound infection, umbilical hernia, displacement of peritoneal drainage, omentum prolapsed through the trocar wound, respiratory infection, intestinal anastomotic fistula, adhesive intestinal obstruction, etc. Clearance of jaundice (CJ) or jaundice free was defined as the total bilirubin level < $1.2 \mathrm{mg} / \mathrm{dL}$. Early CJ or jaundice free was defined as the total bilirubin level $<1.2 \mathrm{mg} / \mathrm{dL}$ within 6 months postoperatively. Incidence of cholangitis was defined as elevated serum bilirubin (> $2.5 \mathrm{mg} / \mathrm{dL})$, leukocytosis with left shift, and normal to acholic stools in a febrile patient $\left(>38.0^{\circ} \mathrm{C}\right)$.

Steroids, intravenous antibiotics and ursodeoxycholic acid were prescribed to all patients. Methylprednisolone was administered intravenously one day postoperatively at a dose of $1 \mathrm{mg} / \mathrm{kg} /$ day for at least 2 weeks. The intravenous antibiotics were administered postoperatively for 2-4 weeks. Patients were followed up regularly until the end of the study.

\section{Statistical analysis}

The software applied for statistical calculation was IBM SPSS 22.0 for windows 10.0 (SPSS Inc., Chicago, IL, USA). In addition to ORT, the rate of CJ, survival with native liver (SNL) and other demographic data were also analyzed. The differences of SNL among the two groups were analyzed by the Kaplan-Meier Method with endpoints of death or liver transplantation and compared by using the log-rank test. Student's $t$-test and chi-squared were used to analyze continuous variables and qualitative variables, respectively. $P$-values less than 0.05 were considered to be statistically significant.

\section{Cumulative sum analysis of operative time}

Cumulative sum (CUSUM) is the accumulated total difference between each data point and the mean of all data points for a particular metric. CUSUM analysis was well suited to and widely employed in the assessment of new technical skills [8-10]. CUSUM analysis was performed starting with the earliest surgical date. All patients were ordered chronologically. The difference between ORT of each of the 100 cases and the mean ORT of all cases (M-ORT) was then obtained. The CUSUM of ORT was obtained by adding up the calculated difference from the overall mean, starting with the first case to the next cumulatively. If the ORT for a case is more than M-ORT, the addition to the running value of CUSUM of ORT is a positive number (upwards slope on the graph). Conversely, it is a negative number if the ORT for a case is less than M-ORT (downwards slope). This cumulative process is sustained until CUSUM of ORT for the last case is calculated as zero. This allows for a graphical representation of the learning curve, which can be calculated and plotted with the software of Matlab 2016a for Windows 10.0 (Mathworks, Natick, MA).

\section{Results}

\section{Perioperative outcomes for the entire cohort}

Table 1 demonstrates perioperative parameters for the entire cohort of patients $(n=100)$. All included cases were non-syndromic BA. The Type III, II and cyst were 97, 2 and $1 \%$, respectively. A total of $64.0 \%$ were female. The median age of the patients was 82.8 days. The median weight was $4.4 \mathrm{~kg}$. The median time of ORT, oral feeding initiated after operation and length of hospital stay was $274 \mathrm{~min}, 4.2$ days and 23.6 days, respectively. The rate of conversion from LKPE to OKPE, intraoperative transfusion and any 
Table 1 Demographic data of patients of two groups with BA

\begin{tabular}{|c|c|c|c|c|}
\hline & $\begin{array}{l}\text { Total } \\
n=100\end{array}$ & $\begin{array}{l}\text { Group A } \\
n=50\end{array}$ & $\begin{array}{l}\text { Group B } \\
n=50\end{array}$ & $P$ \\
\hline Age, (d) & $82.8 \pm 8.4$ & $81.4 \pm 6.4$ & $84.5 \pm 9.9$ & 0.51 \\
\hline Female, n (\%) & $64 / 100(64 \%)$ & $31 / 50(62 \%)$ & $33 / 50(66 \%)$ & 0.68 \\
\hline Weight, (kg) & $4.4 \pm 0.4$ & $4.4 \pm 0.3$ & $4.4 \pm 0.4$ & 0.49 \\
\hline Type (III), n (\%) & 97/100 (97\%) & $49 / 50(98 \%)$ & $48 / 50(96 \%)$ & 0.60 \\
\hline Type (II), n (\%) & $2 / 100(2 \%)$ & $1 / 50(2 \%)$ & $1 / 50(2 \%)$ & 1.0 \\
\hline Type (cyst), n (\%) & $1 / 100(1 \%)$ & 0 & $1 / 60(1.7 \%)$ & - \\
\hline ORT (min) & $274.2 \pm 55.4$ & $316.3 \pm 41.6$ & $232.2 \pm 29.3$ & $<0.01$ \\
\hline Conversion rate, $\mathrm{n}(\%)$ & $11 / 100(11 \%)$ & $10 / 50(20 \%)$ & $1 / 50(2 \%)$ & $<0.01$ \\
\hline Intraoperative transfusion, n (\%) & $26 / 100(26 \%)$ & $16 / 50(32 \%)$ & $10 / 50(20 \%)$ & 0.17 \\
\hline Any perioperative complications, n (\%) & $16 / 100(16 \%)$ & $12 / 50(24 \%)$ & $4 / 50(8 \%)$ & $<0.05$ \\
\hline Mortality, n (\%) & 0 & 0 & 0 & 1.0 \\
\hline Time of oral feeding initiated after operation (d) & $4.2 \pm 1.5$ & $3.7 \pm 1.3$ & $4.6 \pm 1.6$ & 0.43 \\
\hline Length of hospital stay (d) & $23.6 \pm 4.3$ & $22.6 \pm 3.8$ & $24.5 \pm 4.5$ & 0.23 \\
\hline Time of follow-up(month) & $67.2 \pm 12.1$ & $76.7 \pm 8.9$ & $57.5 \pm 5.5$ & $<0.05$ \\
\hline Cholangitis rate, $\mathrm{n}(\%)^{\mathrm{a}}$ & $66 / 100(66 \%)$ & $39 / 50(78.0 \%)$ & $27 / 50(54.0 \%)$ & $<0.01$ \\
\hline CJ rate, n (\%) & $66 / 100(66 \%)$ & $28 / 50(56.0 \%)$ & $38 / 50(74.0 \%)$ & $<0.05$ \\
\hline 1-year SNL rate, n (\%) & $74 / 100(74 \%)$ & $66.0 \%(33 / 50)$ & $82.0 \%(41 / 50)$ & - \\
\hline 3-year SNL rate, n (\%) & $59 / 100(59 \%)$ & $54.0 \%(27 / 50)$ & $64.0 \%(32 / 50)$ & - \\
\hline 5-year SNL rate, n (\%) & 45/100 (45\%) & $36.0 \%(18 / 50)$ & $54.0 \%(27 / 50)$ & - \\
\hline
\end{tabular}

${ }^{a}$ Within one year after operation;

$B A$ : biliary atresia; $C J$ clearance of jaundice; ORT operative time; SNL survival with native liver

perioperative complications was 11,26 and $16 \%$. No patients died during the perioperative period. After a median time of 67.2 months follow-up, the rates of cholangitis and CJ were 66 and 62\%, respectively. The 1-year, 3-year and 5 -year SNL rate were 66,49 and $34 \%$, respectively.
Identification of learning curve based on CUSUM of ORT CUSUM revealed ORT as the one of variables to exhibit improvement across all the patients, whose results of ORT were divided into 10 groups chronologically (Fig. 1). We found that ORT was the only variable to exhibit

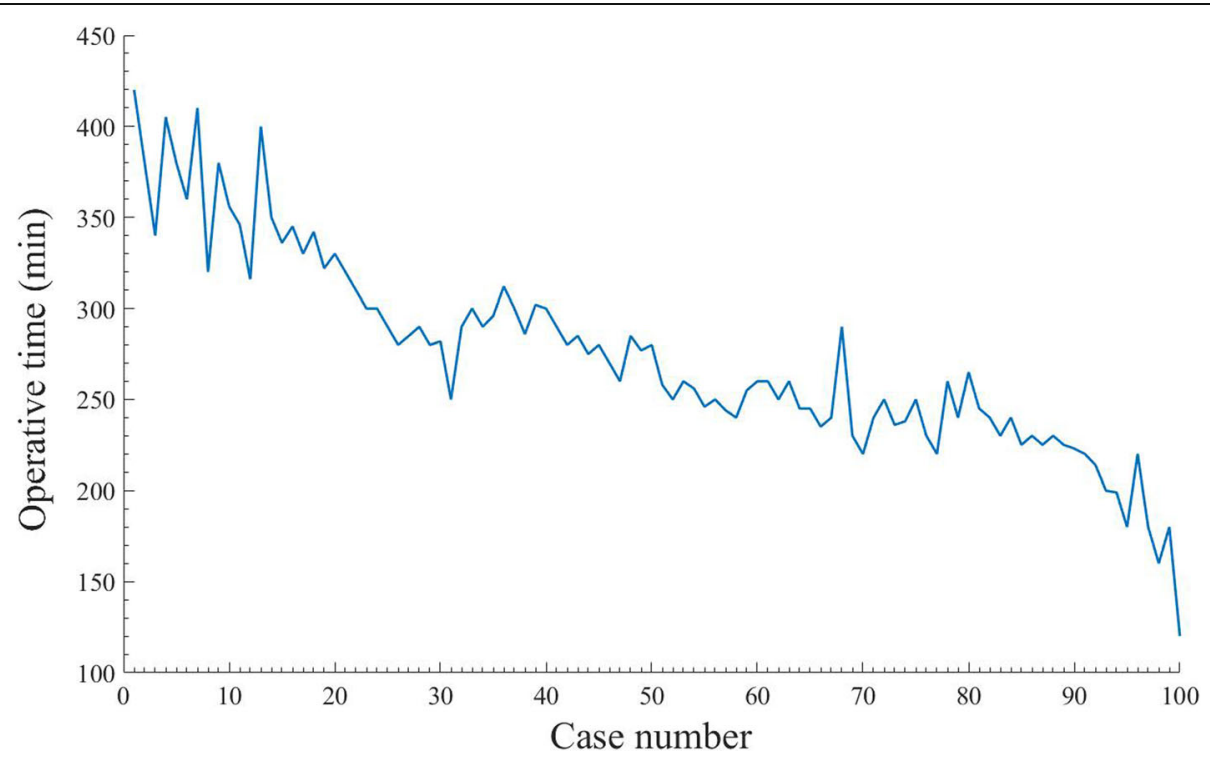

Fig. 1 Decreasing variance from the mean operative time (ORT) with the increasing experience in laparoscopic Kasai portoenterostomy (LKPE) 
improvement across the 10 groups (data not shown). A significant reduction in ORT was displayed after the first 50 cases (from $316.3 \mathrm{~min}$ to $232.2 \mathrm{~min} ; P<0.01$ ) (Fig. 2). These findings confirmed that the two distinct phases of the learning curve in the first 50 cases, followed by a predominantly downward slope. Patients were stratified into 2 groups of 50 patients, with chronological order defining early and late experience. Furthermore, the ORTs beyond the learning curve continued to improve gradually.

\section{Perioperative outcomes in relation to the learning curve}

Based on the identification of the ORT learning curve of 50 cases, an analysis of perioperative outcomes comparing those of in the learning curve cohort (first 50 cases were assigned to 'early experience' or group A), with those in the later experience (post-learning curve cohort: cases 51-100 were assigned to group B) was performed. The two groups were homogeneous with respect to age, female ratio, weight, and type of BA of patients (Table 1 ). The conversion rate from LKPE to OKPE in group $\mathrm{A}$ was much higher than that in group $\mathrm{B}(P<0.01)$. The perioperative complications in group A were more common than that in group B $(P<0.05)$. When compared between the both groups, the rate of intraoperative transfusion, the median time of oral feeding initiated after operation, and the length of hospital stay was not different respectively. The postoperative cholangitis in Group B was significant lower than that in Group A ( $P$ $<0.01)$. In contrast, the $C J$ rate in Group B was significant higher than that in Group A $(P<0.05)$. The median time of follow up was 76.7 months in Group A and 57.5 months in Group B, respectively. The Kaplan-Meier method analysis showed that SNL rate in Group A was significant difference when compared with that in Group B $(P<0.05)$ (Fig. 3).

\section{Discussion}

The adaptations of new technologies are challenges particularly in the practice of medicine [11-15]. There is evidence that the effect of experience is clearly cumulative and can be carried over to later performance [11$18]$. With the aim to better understanding of surgeons' performance during our experience with LKPE, we used CUSUM technology, with combination of CJ and SNL rates, to identify the number of cases that are necessary to achieve the competence of LKPE. These efforts could facilitate more effective training and improve the results of LKPE.

The CUSUM technique is widely used to analyze the learning curve for surgical procedure $[11,12]$ and transforms raw data into running total data deviation from their group mean, enabling investigators to visualize the milestone of learning curve. In this analysis, CUSUM yielded a parabolic curve showing two distinct phases from which correlates of the LKPE learning curve can be assessed. The median ORTs during the first and second phases were $316.3 \mathrm{~min}$ and $232.2 \mathrm{~min}$, respectively. The first phase of steep CUSUM of ORT rapidly rise at case $1 \sim 20$, with a relative stabilization at case $20 \sim 50$, can be attributed to increased familiarity with the 'basics' of the performance. These include the optimal port placement,

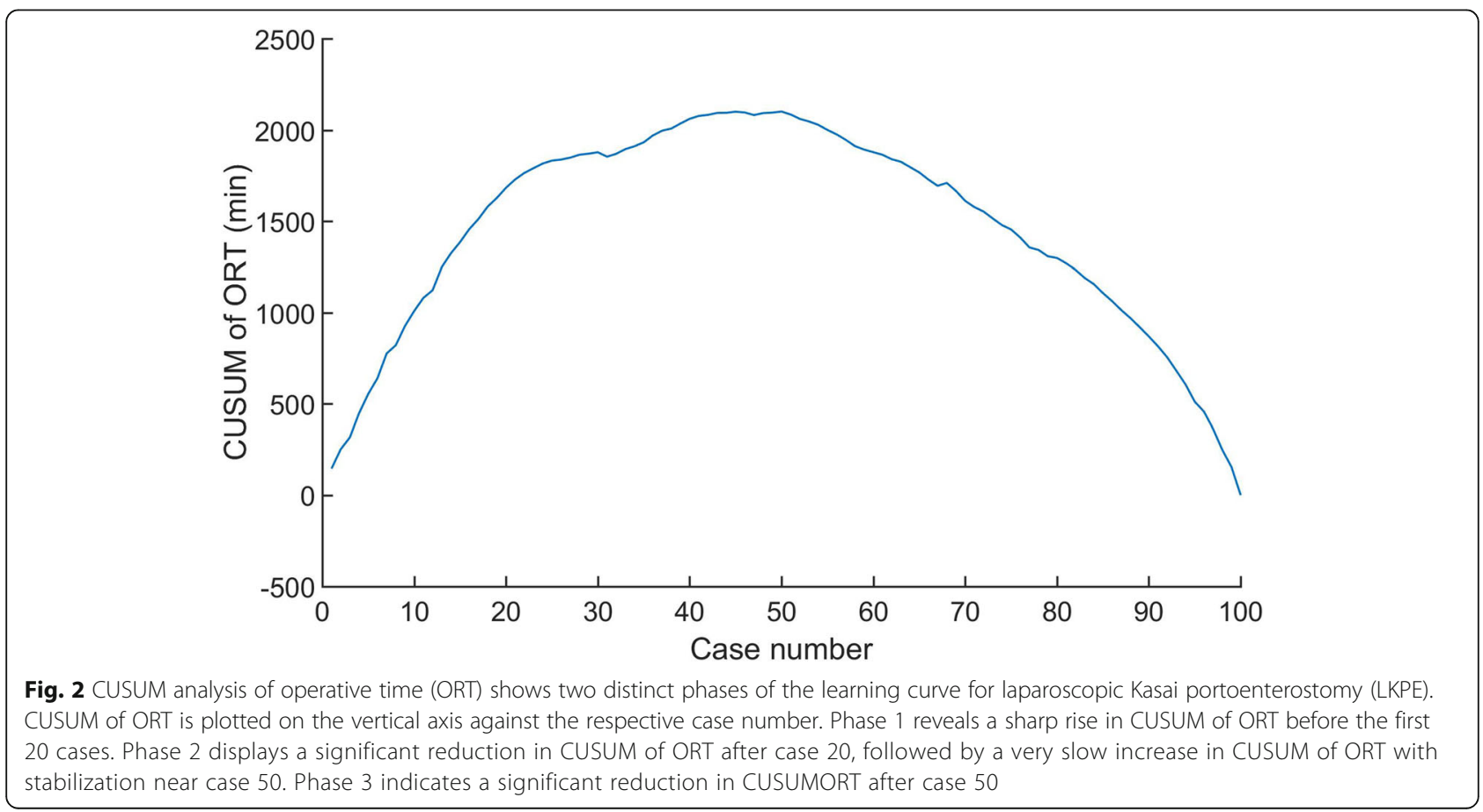




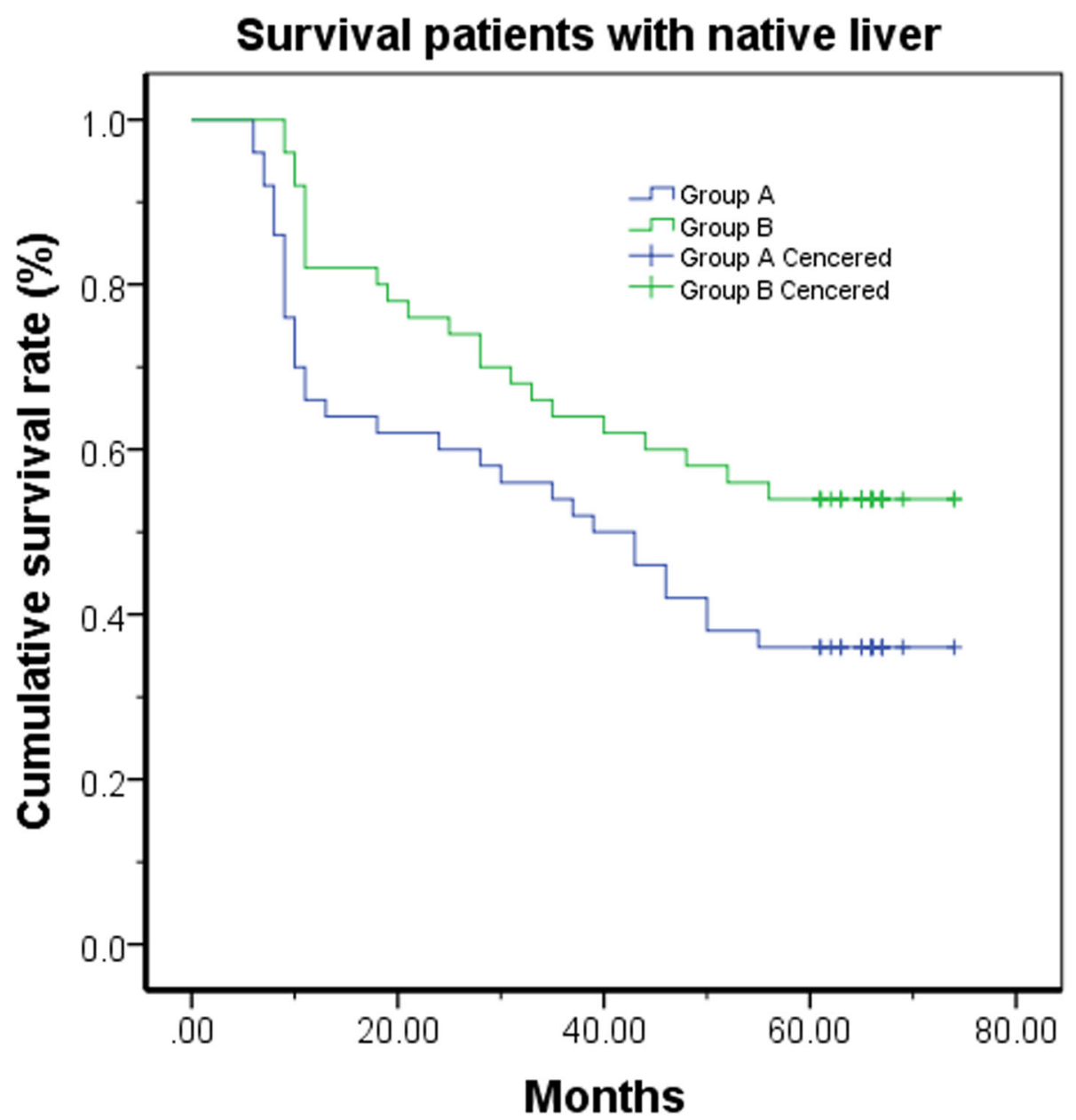

Fig. 3 Kaplan-Meier analysis shows that the survival of patients with native livers (SNL) is statistically significant different between Group A and Group B $(P<0.05)$

the full exposure of porta hepatis, the initial improvement of skills in dissection, the hemostasis, and the portoenterostomy. The phase 2 after case 50 is likely to represent the steep learning curve that reflects the surgeon's development of ability.

As shown, patients in both groups were similar in terms of demographics, weight, and type of BA, suggesting that minimal selection bias was present to influence our results. The conversion rate from LKPE to OKPE, any perioperative complications rate, and cholangitis rate was 20,24 and $78 \%$ in group A, each of which was significant higher than that observed in group $\mathrm{B}$. In addition, the $\mathrm{CJ}$ rate and $\mathrm{SNL}$ rate in group A were worse than that in group B. All perioperative results of both groups mentioned above are highly consistent with the learning curve of LKPE, which was plotted by the cumulative calculation of CUSUM of ORT. A possible explanation for these progression observed in the late-experience period is that the surgeons became more comfortable with postoperative care as they gained experience with the operation.

Interestingly, we observed similar rates of rate of intraoperative transfusion. Although perioperative complications rate increased substantially in group $B$, this finding did not translate into an overall prolonged hospital length of stay. We found that the median time of oral feeding initiated after operation and the length of hospital stay was not different between two groups.

The 50 cases of learning curve in our study was more than that reported by $\mathrm{Li}$ et al. [19]. The authors drew the conclusion that without the usage of Cumulative sum analysis for ORT and Kaplan-Meier analysis for SNL. Nevertheless, we agree with Li that LKPE is really a demanding procedure, especially for novices and those in hospitals with low caseload of BA. In addition, the 50 cases of learning curve of LKPE is more than that of laparoscopic procedure for choledochal cyst reported by Diao's (35 cases) [20] and that of LPCC reported by Zhe's (37 cases) [21]. The younger age at operation, the 
poor exposure of porta hepatis, the difficult assessment for level of dissecting biliary fibrous cone, the challenging procedure for portoenterostomy, all of which may contribute to the "long" learning curve of LKPE.

The age at operation from 1 month to 4 months in our study means that the peritoneal space for manipulation of LKPE is limited. Usually, the full exposure of porta hepatis is often difficult, especially during the initial phase of learning curve. We found that the porta hepatis could be totally displayed by suspension of the segment IV only with percutaneous transhepatic suture. Under the magnification of laparoscopy, the view of operative field can be shown clearly and stably on the screen. Furthermore, the proper level of resecting the bilious fibrous cone is hard to be reached under laparoscopy. Our experiences are that the fibrous cone should be dissected between the first bifurcation of portal vein, and gradually pushed deeper until the leakage or oozing of bile-like liquid is found on the surface of the fibrous stump. Therefore, the base of the fibrous cone would be kept intact. When the portoenterostomy is undertaken, the seams of the anastomoses should be kept as far as possible from the surface of the stump. In addition, the rigorous steroids and prophylactic antibiotic treatment for cholangitis after operation is also necessary for achievement for better long-term results.

Although the outcomes of LKPE are not satisfied with OKPE in some reports $[6,22,23]$, our recent study revealed that the 3 -year and 5 year SNL rates after LKPE were not different compared to those after OKPE. Because BA is a rare disease, the experiences of LKPE are incapable to be cumulated to proficiency in a short time, especially in hospitals or centers with the low caseload of BA. However, the learning curve of LKPE can be shortened by training in the animal experiments, the training devices, such as laparoscopic box and the virtual reality laparoscopic simulator.

Our study has several limitations. Firstly, as a retrospective review, nearly all cases are typeIII nonsyndromic BA, which may not fully represent the entire population of BA. Most patients of syndromic BA in our hospital were given up by their parents, which could have an impact on the learning curve of LKPE. Secondly, the learning curve may actually be shorter than 50 cases for surgeons already experienced in some facets of laparoscopic surgery. The staffs with prior laparoscopic experience and training have been able to climb this learning curve much more rapidly, especially that there are many cases of BA performed each year. Thirdly, the present learning curve analysis is based mainly on ORT, CJ and SNL, which probably failed to display constant significant improvements as a result of small sample sizes.

\section{Conclusions}

In conclusion, the current study identifies the learning curve of LKPE to be approximately 50 cases performed by one team of surgeons with some laparoscopic experiences. The learning curve of LKPE is perhaps more pronounced than many other advanced laparoscopic procedures. The mentorship and dedicated training are likely to contribute to the significant shortening of the learning curve of LKPE in future adopters.

\section{Abbreviations \\ BA: Biliary atresia; CJ: Clearance of jaundice; CUSUM: Cumulative sum; LKPE: Laparoscopic Kasai portoenterostomy; OKPE: Open Kasai portoenterostomy; ORT: Operative time; SNL: Survival with native liver}

\section{Acknowledgements}

We thank the parents of our patient for their co-operation and support, and for providing consent for publication. We also thank the study and hospital nurses for their assistance with the management of the patients.

\section{Funding}

This work was supported by grants from the National Natural Science Foundation of China (Grant No: 81401606 and 81400862) and the Science Foundation for Excellent Youth Scholars of Sichuan University (Grant No: 2015SU04A15)

\section{Availability of data and materials}

The datasets used and/or analyzed during the current study available from the corresponding author on reasonable request.

\section{Authors' contributions}

$Y J, K Y Y, X P Z, S Y C$ and ZCX were involved in the clinical management of these patients and collected clinical details of this study. ZCX reviewed the literature, and drafted the manuscript. YJ reviewed the manuscript. All authors read and approved the final manuscript.

\section{Ethics approval and consent to participate}

The study was approved by the Ethics Committee of the West China Hospital of Sichuan University. Written informed consent was obtained from the patients' parents, according to the provisions of the Declaration of Helsinki.

\section{Consent for publication}

Written informed consent for publication this study was obtained from the patients' parents. Copies of the signed informed consent forms are available for review by the Series Editor of BMC Pediatrics.

\section{Competing interests}

The authors declare that they have no competing interests, either financial or non-financial, that could be perceived as prejudicing the impartiality of the research reported.

\section{Publisher's Note}

Springer Nature remains neutral with regard to jurisdictional claims in published maps and institutional affiliations.

\section{Author details \\ ${ }^{1}$ Department of Pediatric Surgery, West China Hospital of Sichuan University, Chengdu, Sichuan, \#37 Guo-Xue-Xiang, Chengdu 610041, China. ${ }^{2}$ Pediatric Intensive Care Unit, Department of Critical Care Medicine, West China Hospital of Sichuan University, Chengdu 610041, China.}

Received: 14 August 2018 Accepted: 8 November 2018

Published online: 26 November 2018

\section{References}

1. Esteves E, Clemente Neto E, Ottaiano Neto M, Devanir J Jr, Esteves Pereira R. Laparoscopic Kasai portoenterostomy for biliary atresia. Pediatr Surg Int. 2002;18(8):737-40. https://doi.org/10.1007/s00383-002-0791-6. 
2. Davenport M, Ure BM, Petersen C, Kobayashi H. Surgery for biliary atresia--is there a European consensus? Eur J Pediatr Surg. 2007;17(3):180-3. https:// doi.org/10.1055/s-2007-965147.

3. Wong KK, Chung PH, Chan KL, Fan ST, Tam PK. Should open Kasai portoenterostomy be performed for biliary atresia in the era of laparoscopy? Pediatr Surg Int. 2008;24(8):931-3. https://doi.org/10.1007/ s00383-008-2190-0.

4. Ure BM, Kuebler JF, Schukfeh N, Engelmann C, Dingemann J, Petersen C. Survival with the native liver after laparoscopic versus conventional Kasai portoenterostomy in infants with biliary atresia: a prospective trial. Ann Surg. 2011;253(4):826-30. https://doi.org/10.1097/SLA. 0b013e318211d7d8.

5. Wada M, Nakamura H, Koga H, Miyano G, Lane GJ, Okazaki T, Urao M, Murakami H, Kasahara M, Sakamoto S, Ishizaki Y, Kawasaki S, Yamataka A. Experience of treating biliary atresia with three types of portoenterostomy at a single institution: extended, modified Kasai, and laparoscopic modified Kasai. Pediatr Surg Int. 2014;30(9):863-70. https://doi.org/10.1007/s00383014-3551-5.

6. Sun X, Diao M, Wu X, Cheng W, Ye M, Li L. A prospective study comparing laparoscopic and conventional Kasai portoenterostomy in children with biliary atresia. J Pediatr Surg. 2016;51(3):374-8. https://doi.org/10.1016/j. jpedsurg.2015.10.045.

7. Li Y, Xiang B, Wu Y, Wang C, Wang Q, Zhao Y, Chen S, Ji Y, Xu Z. Mediumterm outcome of laparoscopic Kasai Portoenterostomy for biliary atresia with 49 cases. J Pediatr Gastroenterol Nutr. 2018;66(6):857-60. https://doi. org/10.1097/MPG.00000000000001934.

8. Melich G, Hong YK, Kim J, Hur H, Baik SH, Kim NK, Sender Liberman A, Min BS. Simultaneous development of laparoscopy and robotics provides acceptable perioperative outcomes and shows robotics to have a faster learning curve and to be overall faster in rectal cancer surgery: analysis of novice MIS surgeon learning curves. Surg Endosc. 2015;29(3):558-68. https:// doi.org/10.1007/s00464-014-3698-0.

9. Park EJ, Kim CW, Cho MS, Baik SH, Kim DW, Min BS, Lee KY, Kim NK. Multidimensional analyses of the learning curve of robotic low anterior resection for rectal cancer: 3-phase learning process comparison. Surg Endosc. 2014;28(10):2821-31. https://doi.org/10.1007/ s00464-014-3569-8.

10. Hu Y, Jolissaint JS, Ramirez A, Gordon R, Yang Z, Sawyer RG. Cumulative sum: a proficiency metric for basic endoscopic training. I Surg Res. 2014; 192(1):62-7. https://doi.org/10.1016/j.jss.2014.05.056.

11. Subramonian K, Muir G. The 'learning curve' in surgery: what is it, how do we measure it and can we influence it? BJU Int. 2004;93(9):1173-4. https:// doi.org/10.1111/j.1464-410X.2004.04891.x.

12. Guillonneau BD. The learning curve as a measure of experience. Nat Clin Pract Urol. 2005;2(7):309. https://doi.org/10.1038/ncpuro0249.

13. Aggarwal $R$, Moorthy $K$, Darzi A. Laparoscopic skills training and assessment. Br J Surg. 2004;91(12):1549-58. https://doi.org/10.1002/bjs.4816.

14. Morgenstern L. Warning! Dangerous curve ahead: the learning curve. Surg Innov. 2005;12(1):101-3. https://doi.org/10.1177/155335060501200114.

15. Buchmann P, Dincler S. Learning curve--calculation and value in laparoscopic surgery. Ther Umsch. 2005;62(2):69-75. https://doi.org/10.1024/ 0040-5930.62.2.69

16. Ramsay CR, Grant AM, Wallace SA, Garthwaite PH, Monk AF, Russell IT. Assessment of the learning curve in health technologies. A systematic review. Int J Technol Assess Health Care. 2000;16(4):1095-108.

17. Chaput de Saintonge DM, Vere DW. Why don't doctors use cusums? Lancet. 1974:17(7848):120-1.

18. Wohl $\mathrm{H}$. The cusum plot: its utility in the analysis of clinical data. N Engl J Med. 1977;296(18):1044-5. https://doi.org/10.1056/NEJM197705052961806.

19. Li Z, Ye Y, Wu Z, Wang B. Learning curve analysis of laparoscopic Kasai Portoenterostomy. J Laparoendosc Adv Surg Tech A. 2017;27(9):979-82. https://doi.org/10.1089/lap.2016.0204

20. Diao M, Li L, Cheng W. Laparoscopic versus open roux-en-Y hepatojejunostomy for children with choledochal cysts: intermediate-term follow-up results. Surg Endosc. 2011;25(5):1567-73. https://doi.org/10.1007/ s00464-010-1435-x.

21. Wen Z, Liang H, Liang J, Liang Q, Xia H. Evaluation of the learning curve of laparoscopic choledochal cyst excision and roux-en-Y hepaticojejunostomy in children: CUSUM analysis of a single surgeon's experience. Surg Endosc. 2017;31(2):778-87. https://doi.org/10.1007/ s00464-016-5032-5.
22. Chan KW, Lee KH, Tsui SY, Wong YS, Pang KY, Mou JW, Tam YH Laparoscopic versus open Kasai portoenterostomy in infant with biliary atresia: a retrospective review on the 5 -year native liver survival. Pediatr Surg Int. 2012;28(1 1):1109-13. https://doi.org/10.1007/s00383-012-3172-9.

23. Hussain MH, Alizai N, Patel B. Outcomes of laparoscopic Kasai portoenterostomy for biliary atresia: a systematic review. J Pediatr Surg. 2017;52(2):264-7. https://doi.org/10.1016/j.jpedsurg.2016.11.022.
Ready to submit your research? Choose BMC and benefit from:

- fast, convenient online submission

- thorough peer review by experienced researchers in your field

- rapid publication on acceptance

- support for research data, including large and complex data types

- gold Open Access which fosters wider collaboration and increased citations

- maximum visibility for your research: over $100 \mathrm{M}$ website views per year

At BMC, research is always in progress.

Learn more biomedcentral.com/submissions 